\title{
A large-scale genome-wide association and meta- analysis identified four novel susceptibility loci for leprosy
}

Zhenzhen Wang ${ }^{1,2, \star}$, Yonghu Sun ${ }^{1,2, \star}, X^{\prime}$ an Fu' ${ }^{1,2,3, *}$, Gongqi $\mathrm{Yu}^{1,2,4, \star}$, Chuan Wang ${ }^{1,2}$, Fangfang Bao ${ }^{1,2}$, Zhenhua Yue ${ }^{1,2,3}$, Jianke $\mathrm{Li}^{1,2,5}$, Lele Sun ${ }^{1,2}$, Astrid Irwanto ${ }^{6}$, Yongxiang $\mathrm{Yu}^{1,2}$, Mingfei Chen ${ }^{1,2}$, Zihao $\mathrm{Mi}^{1,2}$,

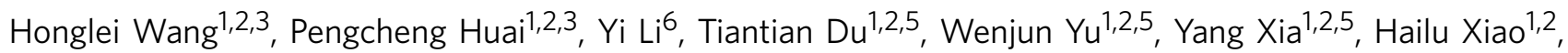
Jiabao You ${ }^{1,2}$, Jinghui $\mathrm{Li}^{1,2}$, Qing Yang ${ }^{1,5}$, Na Wang1,2,3, Panpan Shang ${ }^{1,2}$, Guiye Niu1,2, Xiaojun Chi ${ }^{1,2,5}$, Xiuhuan Wang ${ }^{1,2,5}$, Jing Cao ${ }^{1,2,3}$, Xiujun Cheng ${ }^{1,2,3}$, Hong Liu'1,2,5, Jianjun Liu 6 \& Furen Zhang 1,2,3,4,5,7

Leprosy, a chronic infectious disease, results from the uncultivable pathogen Mycobacterium leprae (M. leprae), and usually progresses to peripheral neuropathy and permanent progressive deformity if not treated. Previously published genetic studies have identified 18 gene/loci significantly associated with leprosy at the genome-wide significant level. However as a complex disease, only a small proportion of leprosy risk could be explained by those gene/loci. To further identify more susceptibility gene/loci, we hereby performed a threestage GWAS comprising 8,156 leprosy patients and 15,610 controls of Chinese ancestry. Four novel loci were identified including rs6807915 on 3p25.2 $\left(P=1.94 \times 10^{-8}, \mathrm{OR}=0.89\right)$, rs4720118 on 7p14.3 $\left(P=3.85 \times 10^{-10}, \mathrm{OR}=1.16\right)$, rs55894533 on 8p23.1 $(P=5.07 \times 10$ $-11, \mathrm{OR}=1.15)$ and $\mathrm{rs} 10100465$ on $8 \mathrm{q} 24.11\left(P=2.85 \times 10^{-11}, \mathrm{OR}=0.85\right)$. Altogether, these findings have provided new insight and significantly expanded our understanding of the genetic basis of leprosy.

\footnotetext{
${ }^{1}$ Shandong Provincial Institute of Dermatology and Venereology, Shandong Academy of Medical Sciences, Jinan, Shandong 250000, China. ${ }^{2}$ Shandong Provincial Key Laboratory for Dermatovenereology, Jinan, Shandong 250000, China. ${ }^{3}$ School of Medicine, Shandong University, Jinan, Shandong 250000 , China. ${ }^{4}$ School of Medicine and Life Science, University of Jinan-Shandong Academy of Medical Sciences, Jinan, Shandong 250022, China. 5 Shandong Provincial Hospital for Skin Diseases, Shandong University, Jinan, Shandong 250000, China. ${ }^{6}$ Human Genetics, Genome Institute of Singapore, Singapore 138672, Singapore. ${ }^{7}$ National Clinical Key Project of Dermatology and Venereology, Jinan, Shandong 250000, China. * These authors contributed equally to this work. Correspondence and requests for materials should be addressed to H.L. (email: hongyue2519@hotmail.com) or to F.Z. (email: zhangfuren@hotmail.com).
} 
eprosy, an ancient mycobacterial disease, results from the uncultivable pathogen Mycobacterium leprae, and usually progresses to peripheral neuropathy and permanent progressive deformity if not being treated ${ }^{1}$. The clinical features of leprosy present a five-group spectrum, likely reflecting the interactive outcome between the host immune responses and the pathogen. Since the implementation of multidrug therapy, the prevalence of leprosy has been significantly reduced worldwide. However, due to the occurrence of permanent disabilities and sequelae, leprosy still represents a serious health problem in the developing countries ${ }^{2}$.

The role of host genetic factors in the development of leprosy has been well established through epidemiological and molecular genetic studies. Multiple genes and loci have been discovered as leprosy risk factors, such as PARK2-PACRG (ref. 3), IL10 (ref. 4), VDR (ref. 5), LTA (ref. 6) and HLA-DR (ref. 7), but only a few were replicated. Recently, the understanding of leprosy genetic factors has been remarkably improved by the application of genome-wide association studies (GWASs), which have discovered 18 leprosy associated susceptibility gene/loci ${ }^{8-12}$, most of which are related to immunity and inflammatory responses, providing valuable insights and emphasizing the important role of genetic risk factors in disease development. However, these loci can only explain a small proportion of the disease risk and heritability, indicating that additional genetic risk factors remain to be discovered.

Here, we performed a new GWAS analysis including 1,197 leprosy cases and 1,426 controls by using a population-specific array (Illumina Omni Zhonghua Array). Furthermore, we conducted a three-stage GWAS Meta analysis comprising 8,156 cases and 15,610 controls of Chinese ancestry. We confirmed all the known leprosy susceptibility loci and identified four novel loci on 3p25.2 (SYN2), 7p14.3 (BBS9), 8p23.1 (CTSB) and 8q24.11 (MED30). Altogether, these findings significantly expand the understanding of the disease susceptibility factor and suggest new biological pathways related to leprosy.

\section{Results}

Genome-wide discovery analysis. To discover additional leprosy susceptibility loci, we carried out a large-scale three-stage GWAS analysis of leprosy in Chinese population. The genome-wide discovery analysis (Stage 1) involved two published GWAS dataset of leprosy ${ }^{8,12}$, consisting of leprosy patients and geographically matched controls from northern part (Chinese Han) and southern part of China (Chinese Han and ethnic minorities), details in the Methods. The third sample was a new GWAS data set (GWAS3) of 1,197 leprosy cases and 1,426 controls from northern (Chinese Han) and southern China (Chinese Han) conducted by using Illumina Omni Zhonghua chips with 900,015 single-nucleotide polymorphisms (SNPs).

We performed the genome-wide imputation in the three GWAS data sets seperately, aiming to obtain a more comprehensive genome-widely coverage of genetic variants. The untyped SNPs were imputed by using the multi-ethnic reference panel from the 1000-genome project (March 2012 release, IMPUTE v2). Principal components analysis confirmed that all the samples were Chinese ancestry. Quality control filtering was performed to the imputed datasets as described in the Methods. Finally, we tested the associations of 5,546,030 common SNPs (minor allele frequency $>1 \% ; 258,961$ genotyped, 5,287,069 imputed) in a total number of 2,743 leprosy patients and 3,573 healthy controls. Both the quantile-quantile plots (QQ plots) (Supplementary Fig. 1) and genomic inflation factors $\left(\lambda_{\mathrm{GC}}\right)$ of the genome-wide test statistic (1.026 for GWAS1, 1.019 for North Han of GWAS2, 0.98 for South Han of GWAS2, 1.052 for South Minority of GWAS2, 1.038 for North Han of GWAS3 and 1.066 for South Han of GWAS3) suggested minimal inflation on the population stratification. As shown in Fig. 1, all the 18 reported leprosy susceptibility gene/loci showed significant association in the new meta-analysis. Furthermore, additional suggestive SNPs with $P$ values $<5 \times 10^{-4}$ from association analysis in the new GWAS or combined data sets as described in the method section were observed (Supplementary Fig. 2).

Validation analysis of novel associations. In total, we selected 168 top independent SNPs that met our selection criteria (Methods) for a follow-up genotyping validation analysis (Stage 2) in an independent cohort comprising 1,516 leprosy patients and 1,512 healthy controls of northern Chinese Han.

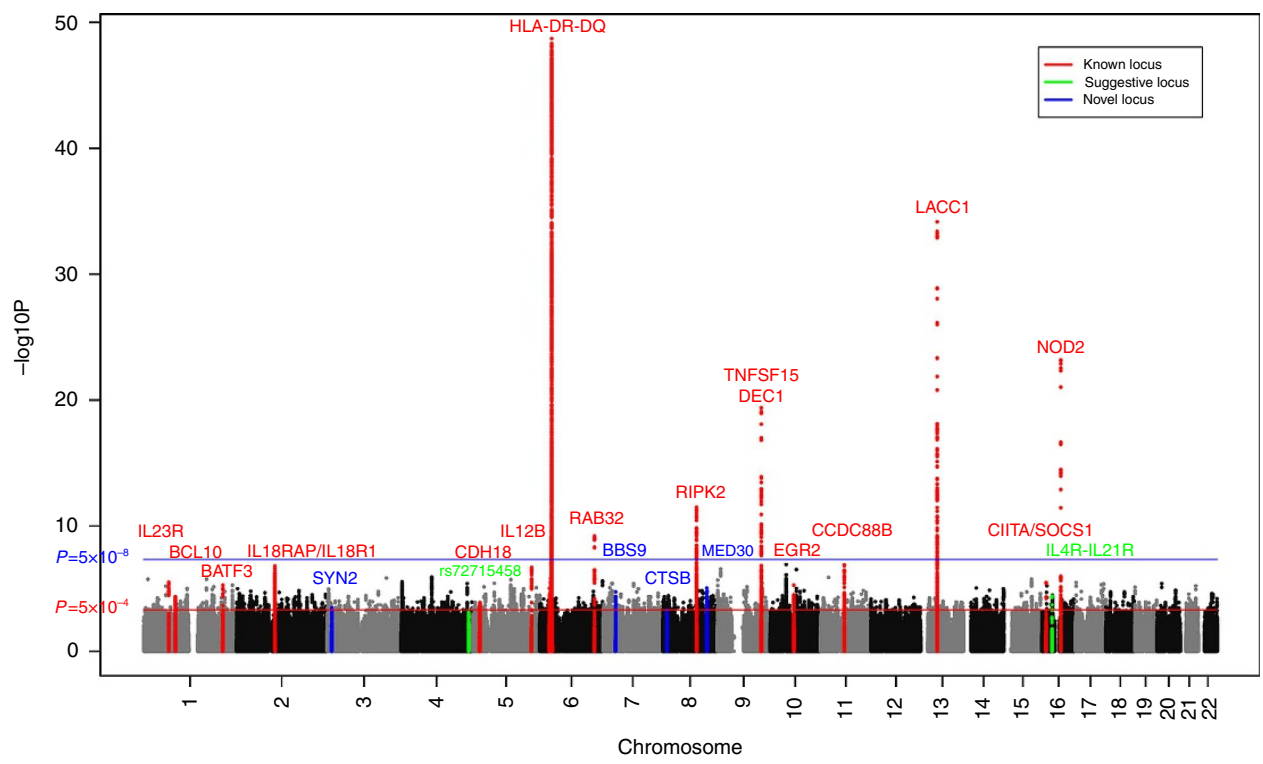

Figure 1 | Chromosomal plot of the genome-wide association analysis. Known loci are defined as loci previously published with genome-wide significance $\left(P<5 \times 10^{-8}\right)$; Suggestive loci are defined as loci with suggestive evidence of association $\left(1 \times 10^{-6}<P<5 \times 10^{-8}\right)$; Novel loci are defined as loci in the current study validated at genome-wide significance $\left(P<5 \times 10^{-8}\right)$. 
Of the 127 successfully genotyped SNPs, we subsequently selected 21 significant SNPs with $P<0.05$ and showing a consistent risk effect across the Stage 1 and 2 analyses for a further validation analysis (Stage 3) in four additional independent sample series from different geographic regions of China, totalling 3,897 cases and 10,525 controls (Supplementary Tables 2 and 3). The combined analysis of Stage 2 and 3 samples revealed significant association at seven SNPs after correction for multiple testing for 127 SNPs.

Totalling 8,156 leprosy patients and 15,610 healthy controls were involved in the joint association analysis (Stage 1,2 and 3) using meta-analysis under a fixed-effects model. Four novel associations were discovered at genome-wide significance $\left(P<5 \times 10^{-8}\right)$, including rs6807915 on $3 \mathrm{p} 25.2\left(P=1.94 \times 10^{-8}, \mathrm{OR}=0.89\right)$, rs4720118 on $7 \mathrm{p} 14.3\left(P=3.85 \times 10^{-10}, \mathrm{OR}=1.16\right), \mathrm{rs} 55894533$ on $8 \mathrm{p} 23.1\left(P=5.07 \times 10^{-11}, \mathrm{OR}=1.15\right)$, and $\mathrm{rs} 10100465$ on 8q24.11 $\left(P=2.85 \times 10^{-11}, \quad \mathrm{OR}=0.85\right) \quad($ Table 1$)$. Two novel suggestive associations were also identified at rs72715458 on $4 \mathrm{q} 34.3$ $\left(P=2.62 \times 10^{-7}, \quad \mathrm{OR}=0.85\right) \quad$ and $\mathrm{rs} 34411505$ on $16 \mathrm{p} 12.1$ $\left(P=5.82 \times 10^{-7}, \mathrm{OR}=0.86\right)$, whose evidence was just below genome-wide significance (Table 1).

Gene prioritization of those novel associations. Most of the novel associations are located within polygenic LD blocks (Fig. 2). To evaluate susceptibility gene candidates within the newly confirmed loci, we performed a gene prioritization based on a differentiated gene expression analysis, which estimates the relevance gene expression between leprosy biopsy and healthy control skin through an unpublished RNA-sequence dataset (27 leprosy biopsy Vs 18 healthy controls) (Fig. 3). Those genes mostly nearby the lead variant were taken into consideration as potentially causal.

The SNP rs6807915 located between SYN2 and PPARG gene, which were significantly down regulated in the lesion of leprosy patients. Although we did not find direct evidence of eQTL for rs6807915, the significant eQTL effect of four highly correlated SNPs $\left(r^{2}>0.9, D^{\prime}>0.9\right.$, Supplementary Table 4$)$ with it suggested that SYN2 might more likely to be the causal gene of 3p25.2. The SNP rs4720118 located in the eighteenth intron of BBS9 gene, which was significantly down regulated in the skin of leprosy patient. It was found that this SNP could significantly regulate the expression of BBS9 in whole blood. The SNP rs55894533 located nearby the $5^{\prime}$ of $C T S B$ gene, which were significantly up regulated in the leprosy patients. Significant eQTL effect of rs55894533 was found in whole blood and fibroblasts for CTSB gene. The SNP rs10100465 located nearby MED30 gene, which was downregulated in the skin of leprosy patients. Further eQTL analysis demonstrated this SNP could regulate the expression of MED30 in whole blood.

Fine mapping of the associations in the MHC region. As expected, the strongest association signal in the meta-analysis was observed within the MHC region. To fine-map and elucidate the signals, we tested the association within the MHC region after imputing untyped SNPs, classical HLA alleles and polymorphic amino acid positions in the discovery data set. The significant associations within the MHC region were discovered to locate within the MHC class II region. HLA-DRB1 ${ }^{\star} 15$ was identified as the most significant risk allele $\left(P=4.21 \times 10^{-44}\right.$; $\left.\mathrm{OR}=2.17\right)$. The effect of all the other associations within the MHC region could be eliminated by conditioning on HLA-DRB1 ${ }^{\star} 15$. The full association results of classical HLA allele were provided in Supplementary Table 5 .

Heritability and enrichment analysis. We investigated the proportion of risk variance and heritability explained by genome- wide SNPs using Genome-wide Complex Trait Analysis (GCTA) method. We estimated the SNP heritability of leprosy at 0.199 (s.e. $=0.01$ ), by using the genotyped autosomal SNPs and assuming the disease prevalence of leprosy as 0.0001 . In total, all the identified Genome-wide significant variants thus far as being robustly associated with leprosy risk explain $\sim 13.53 \%$ on the liability scale (Supplementary Table 6). We conducted the heritability partitioning by tissue and functional category using LD score regression and identified significant enrichment in multiple tissue. The most significant enrichment was found in immune cells (enrichment $=3.74, P=3.2 \times 10^{-8}$ ), suggesting the important role of immunity in the disease aetiology. Region of functional category of genome with the most significant enrichment is transcription start site (TSS, enrichment $=16.3$, $\left.P=2.52 \times 10^{-4}\right)$ (Supplementary Fig. 3).

\section{Discussion}

The current large-scale GWAS meta-analysis of leprosy has several advantages comparing to our previous GWAS analysis. First, we carried out a new Genome-wide genotyping by Illumina Omni Zhonghua Chip, which is designed specific to Chinese Han population and may uncover the population-specific susceptibility loci. Second, multiple risk alleles with small effect size were detected by the increased sample size and improved statistical power. Through the meta-analysis of a total number of 8,156 leprosy patients and 15,610 healthy controls, we have identified four novel associations, all of which can indicate candidate genes within the susceptibility loci, SYN2 on 3p25.2, BBS9 on $7 \mathrm{p} 14.3, C T S B$ on $8 \mathrm{p} 23.1$ and MED30 on 8q24.11, through a differential gene expression and eQTL analysis.

At 3p25.2, we identified a non-coding variant nearby $P P A R G$ and SYN2 that were both significantly down regulated in the lesion of leprosy patients. Further eQTL analysis has suggested that SYN2 might more likely to be the causal gene than PPARG within the 3p25.2 locus. SYN2 encodes neuronal phosphoproteins and belongs to the synapsin gene family, which is found to be associated with the cytoplasmic surface of synaptic vesicles. There have been several publications that support the significant association of SYN2 with type II diabetes ${ }^{13,14}$, interaction with BMI (ref. 15) and schizophrenia ${ }^{16}$. Furthermore, a downregulated gene expression of synapsin 2 was discovered in the prefrontal cortex of schizophrenic patients ${ }^{17}$. Interestingly, the expression SYN2 was also down-regulated in the leprosy biopsy in our study, which may suggest its role in the infection progress of mycobacteria to the nerves.

At $7 \mathrm{p} 14.3$, we identified a non-coding variant within the eighteenth intron of $B B S 9$ gene. It was significantly up regulated in the leprosy biopsy, while its eQTL effect was found in the thyroid and whole blood. The expression of $B B S 9$ could be downregulated by parathyroid hormone in an osteoplastic cell line $e^{18}$ and interrupted in a translocation breakpoint associated with Wilms Tumour ${ }^{19}$. Although we did not find any publication of its role on bacteria infection or autoimmunity, the fact that association and differentiated gene expression of BBS9 gene suggest a potential role in the pathogenesis of leprosy.

At 8p23.1, Cathepsin B(CTSB) were found to be significantly up regulated in the leprosy patients. In addition, eQTL effect of the identified SNP was also observed in whole blood and fibroblasts for CTSB gene. CTSB encodes a lysosomal cysteine protease and has been reported to contribute to the progression and invasion of multiple cancer ${ }^{20,21}$. In the stratum spinosum of human skin, CTSB is found to be presented within vesicles of cellular protrusions forming cell-cell contact sites between keratinocytes ${ }^{22}$. It is also evident that a block in CTSB expression reduced the migration ability of keratinocytes and unobstructed 


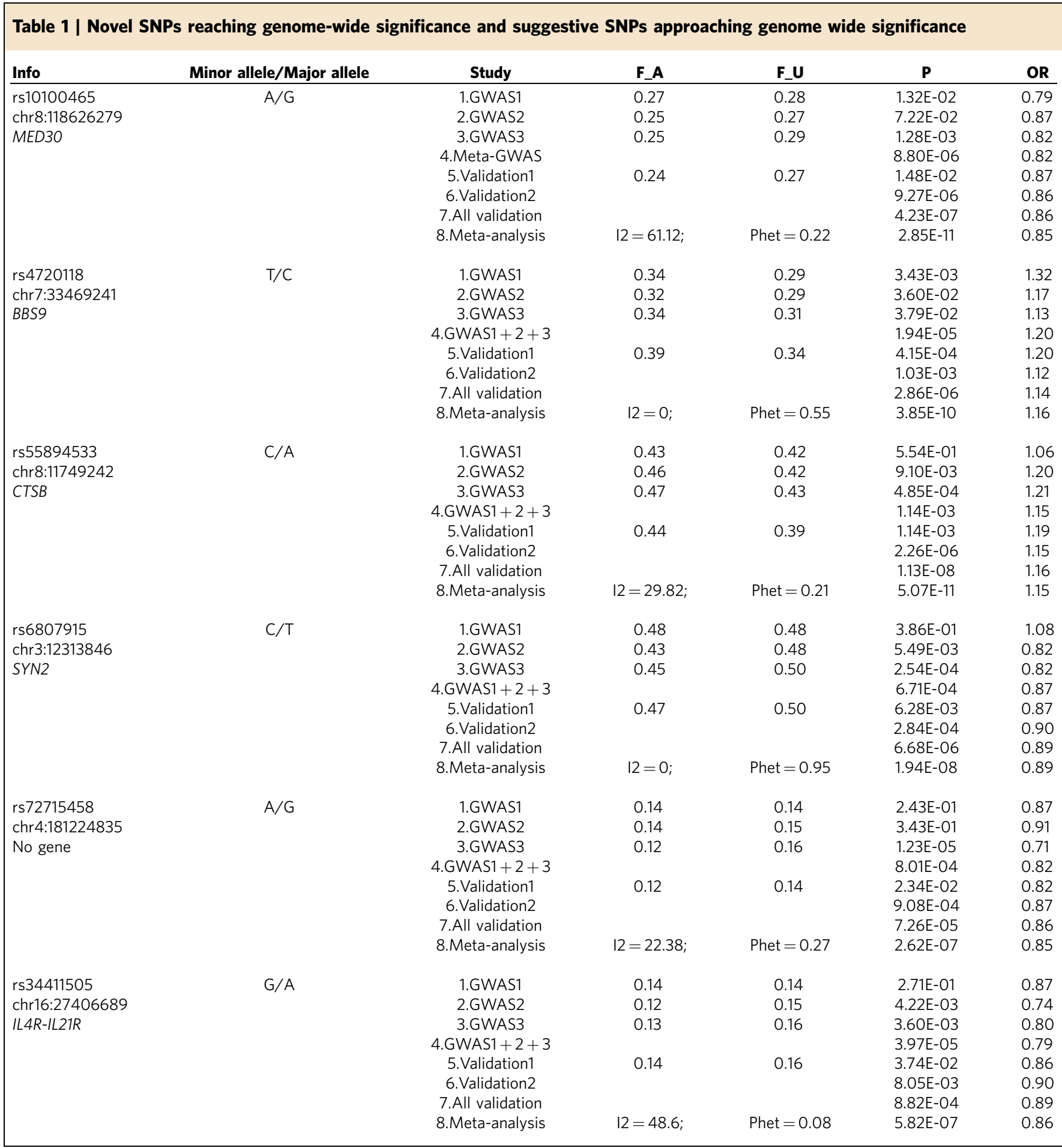

migration of keratinocytes, which could possibly explain the over expression of CTSB in the leprosy lesion. This remains to be further investigated.

At $8 \mathrm{q} 24.11, M E D 30$ was identified as the potential causal gene through robust association and differentiated gene expression. MED30 was previously reported as a suggested association with Kawasaki disease in Han Chinese population ${ }^{23}$.

Besides these four newly identified leprosy susceptibility loci, we noted two additional suggestive associations at $4 \mathrm{q} 34.3$ and $16 \mathrm{p} 12.1$ whose evidences were both just below the genome-wide significance. The first of the two suggestive disease association rs72715458 on $4 \mathrm{q} 34.3$ lies within an intergenic region without any identifiable genes in the LD block. The second suggestive association rs34411505 on 16p12.1 locates between IL4R and $I L 21 R$ whose expressions were both elevated in the leprosy patients. IL4R and IL $21 R$ both encode immunomodulatory cytokine that regulate adaptive immunity responses, which play important role in the leprosy development. In addition, IL-4 and IL-21 could effect on the proliferation and differentiation and subsequently activate B cells ${ }^{24}$.

HLA allele has long been thought to play key role on the development of leprosy. Early studies comparing allele frequencies in leprosy patients and controls have identified associations in both HLA class I gene and class II gene, but results were 
a

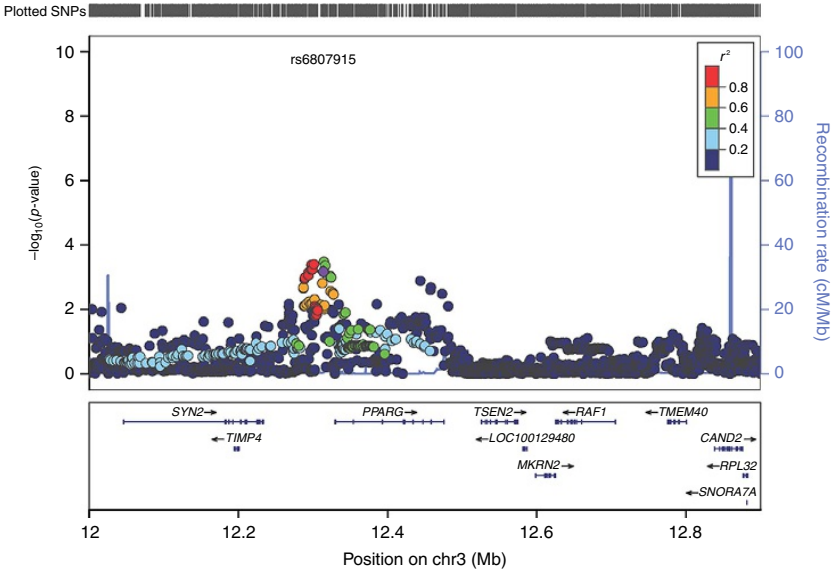

C

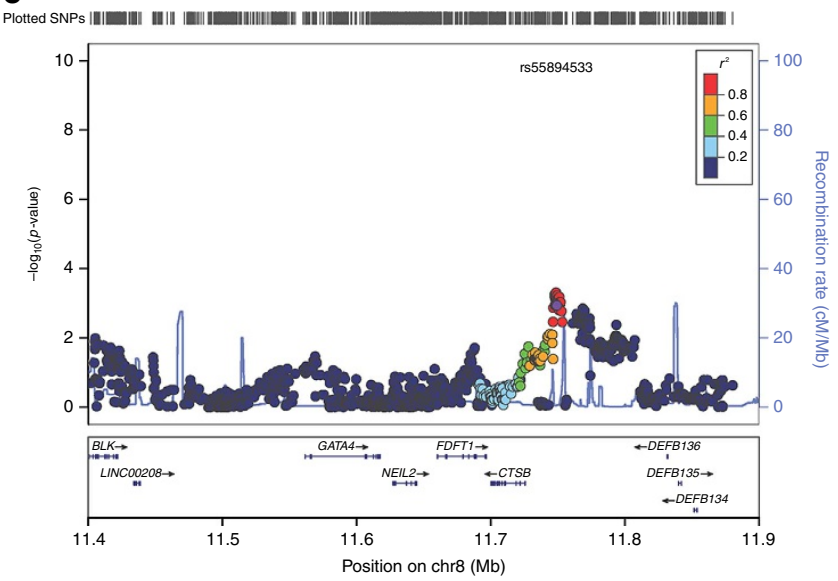

b

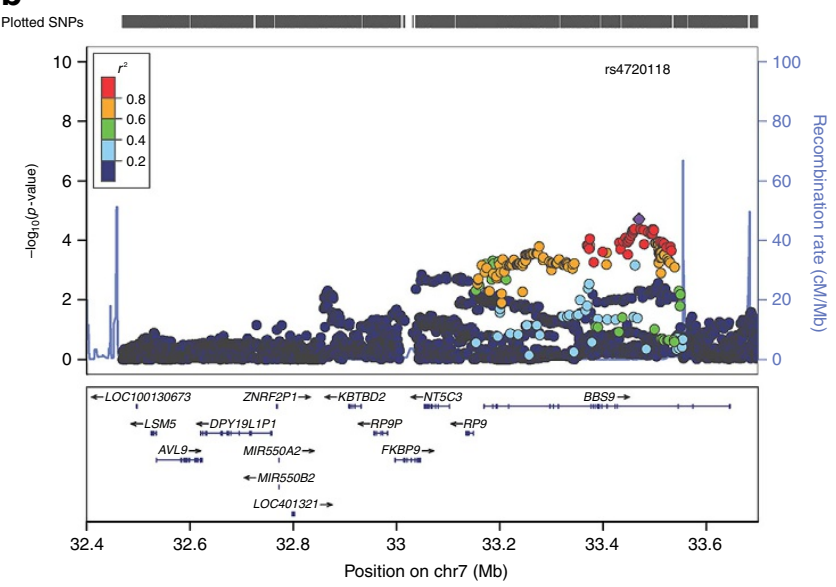

d

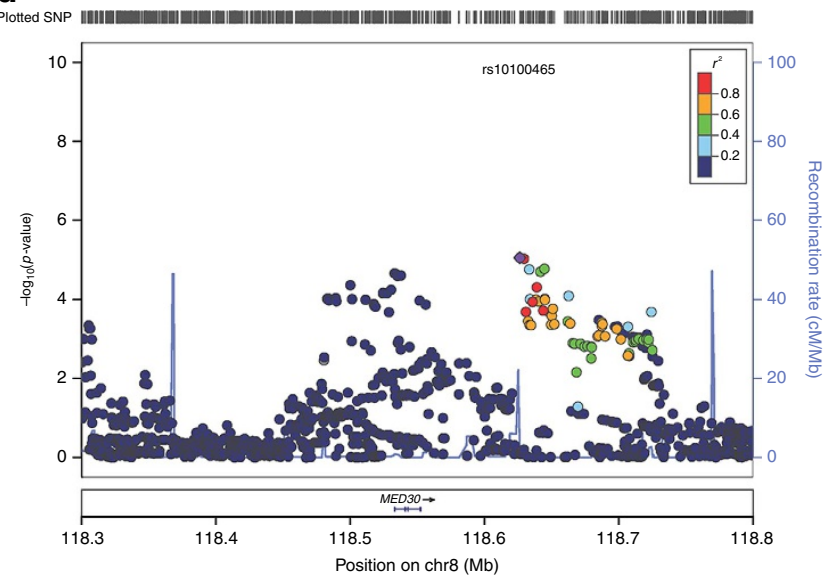

Figure 2 | Recombination plots of the novel loci reaching genome-wide significance. (a) Association at 3p25.2 (between SYN2 and PPARG);

(b) Association at 7p14.3 (within BBS9); (c) Association at 8p23.1 (Nearby CTSB); (d) Association at 8q24.11 (Nearby MED30). SNP labelled in purple is the validated SNP. All coordinates are based on NCBI build 37.

inconsistent. Through HLA imputation, we have refined our previous findings of $\mathrm{HLA}-\mathrm{DRB} 1^{\star} 15$ to a four-digit resolution HLA-DRB $1^{\star} 1501$, which is consistent with the previously HLA association analysis in Indian ${ }^{25,26}$ and Thai $^{27}$ populations. Other previously reported associations, such as protective allele HLA-DRB $1^{\star} 04$ to Brazilian ${ }^{28}$, has been confirmed as suggestive association and similar effect in the current study. HLA-DRB $1^{\star} 1501$ has been reported to associate with several autoimmune disease, such as multiple sclerosis $^{29}$ and SLE (ref. 30), which emphasize the pleotropic role of disease associated gene between infectious disease and autoimmune disease.

Although GWASs have been proven successfully in identifying regions of the genome harbouring variants that contribute to complex diseases, there are several limitations. First, GWAS have generally identified common risk variants with relatively small effect sizes $(\mathrm{OR}<1.5)$, which are lack of clinical translation but can help to identify important biological pathways for diseases. Second, relying on LD-based association analysis, GWAS is not well powered for detecting rare variants that may have larger effect on disease. Third, for most diseases the effects of all the identified susceptibility loci only account for a small proportion of the estimated heritability, even in diseases where extremely large sample size had been analysed, such as leprosy. By using GCTA method and assuming the leprosy disease prevalence as 0.0001 , we estimate the heritability of leprosy attributable to genome-wide SNPs to be 0.199 on the liability scale. This estimate differs from the results from previous genetic epidemiology studies, due to the exclusion of non-additive genetic effects, as well as the effect of gene-environment interaction on leprosy. Finally, the loci identified by GWAS are largely located within non-coding genomic regions, which make it challenging to narrow down causal genes and perform further functional experiments.

Interestingly, all the identified leprosy susceptibility gene/loci at genome-wide significance can only explain $\sim 13.53 \%$ of phenotypic variance on the liability scale, which is about 0.199 of the heritability that can be genome-wide SNPs. By conducting the heritability partitioning of leprosy GWAS by tissue and functional category using LD score regression, we identified the most significant enrichment in immune cells and transcript start site, suggesting the important role of immunoregulatory and non-coding variants, but remain further experimental methods to fine-map the causal variant(s).

In summary, we have conducted the largest Genome-wide meta-analysis study on leprosy in the Chinese population to date. By analysing a total number of 8,156 leprosy patients and 15,610 healthy controls, we identified four novel loci and two suggestive loci, which has added new knowledge on the genetic basis of leprosy susceptibility.

\section{Methods}

Study subjects. We designed a three-stage case-control analysis for this study. All individuals were of Chinese descent and detailed information for each stage is shown in Supplementary Table 1. All the cases and controls were recruited with the 

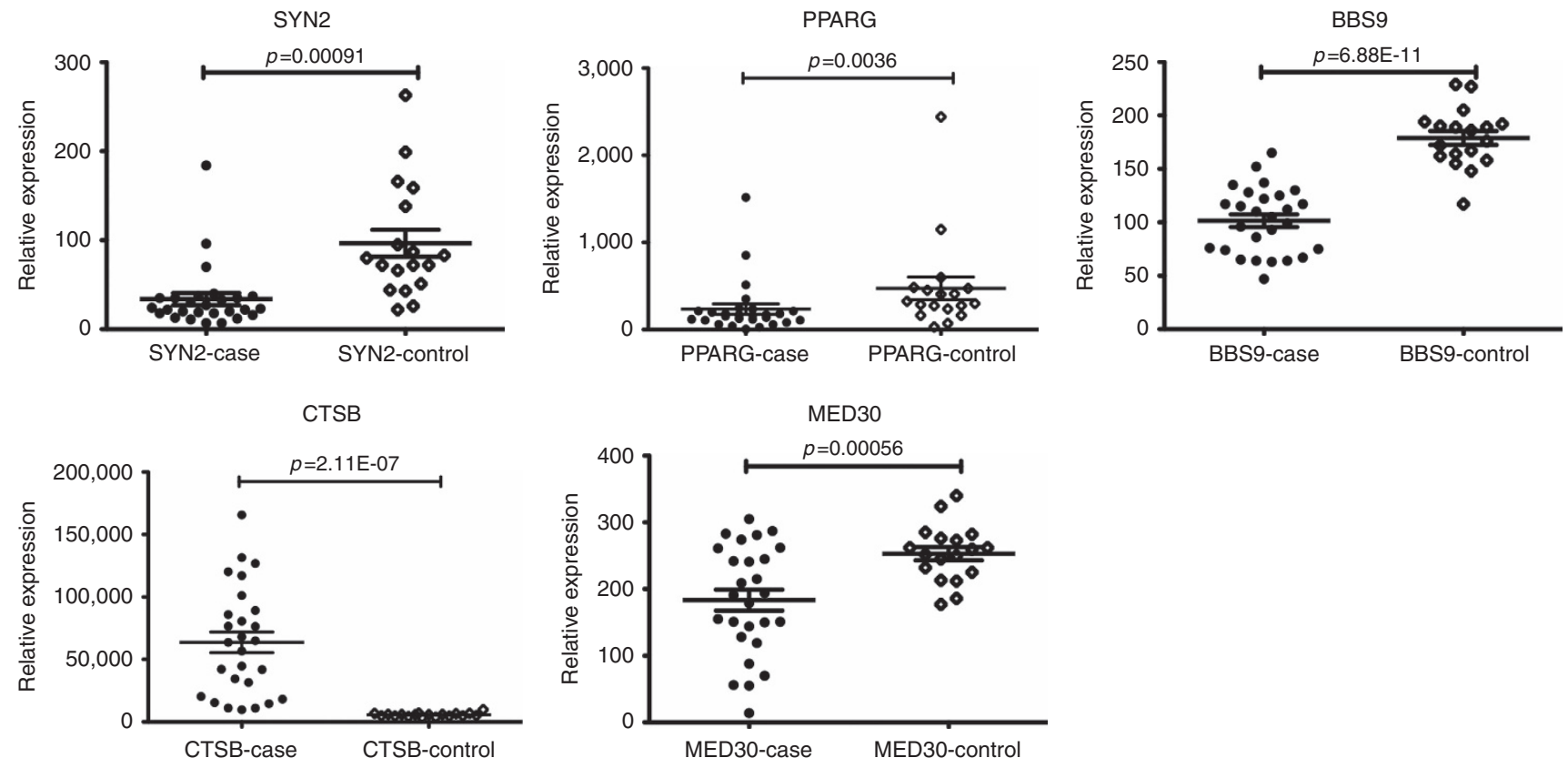

Figure 3 | Relative gene expression of susceptibility genes nearby the lead association. The transcripts of these encompassed genes in the susceptibility region measured by RNA sequence data (SYN2 and PPARG on 3p25.2, BBS9 on 7p14.3, CTSB on 8p23.1 and MED30 on 8q24.11). Statistical significance was calculated by Student's $t$-test comparing leprosy lesion with healthy control. The horizontal line represents significant expression $(P<0.05)$. The dots represent the enroled samples. Data is shown as mean \pm s.d.

same criteria described in our previous studies ${ }^{8-12}$. The institutional review board committees of Shandong Provincial Institute of Dermatology and Venereology, Shandong Academy of Medical Science approved our study.

The discovery study (Stage 1) consisted three independent GWAS datasets. The sample characteristics of the first two datasets, GWAS1 (706 leprosy patients and 1,225 healthy controls) and GWAS2 (842 leprosy patients and 925 healthy controls), were described in our previous publications ${ }^{8-12}$. The third was a new GWAS dataset (GWAS3) comprising 1,353 leprosy patients and 1,651 controls of Chinese Han descent, including 901 patients and 899 controls from northern part of China and 452 patients and 752 controls from southern part of China.

Validation analyses were performed in two independent cohorts (Stage 2 and 3): in stage 2, 1,516 leprosy patients and 1,512 controls from northern part of China were analysed; in stage 3, 3,897 leprosy patients and 10,525 controls were analysed, consisting of 1,666 cases and 8,259 controls from Shandong Province of northern China, 829 cases and 589 controls from Yunnan Province, 496 cases and 799 controls from Guizhou Province and 906 cases and 878 controls from Sichuan Province (Yunnan, Guizhou and Sichuan are all from southeastern part of China). All the samples in stage 2 and 3 are Chinese Han descent. Totalling 5,413 cases and 12,037 controls were used in the validation stages.

\section{Genome-wide genotyping and quality control in the discovery stage. The} genotyping and quality control (QC) procedures of the first (GWAS1) and the second (GWAS2) datasets have been previously described ${ }^{8,12}$. These procedures were conducted on the new (GWAS3) dataset using Illumina Omni Zhonghua chips (Illumina, Inc., San Diego, CA, USA) and standard QC procedures with the following criteria: We excluded all the SNPs with an overall call rate $<95 \%(1,262$ SNPs), minor allele frequency (MAF) $<1 \%(62,840 \mathrm{SNPs})$, Hardy-Weinberg equilibrium (HWE) $P$ value in control subjects $<1.0 \times 10^{-8}(650 \mathrm{SNP})$. We also excluded all the copy number variations (CNVs), intensity-only SNPs and SNPs located in the idiochromosome (a total of 27,754 SNPs) and SNPs with undetermined clusters (three SNPs). Finally, we used 258,961 overlapped genotyped SNPs in the first three independent datasets for imputation and association analysis.

We conducted sample QC in all individuals of GWAS1, 2 and 3. Those with call rate $<96 \%$ (8 samples) were excluded first. The potential genetic relatedness of all successfully genotyped samples was estimated on the basis of pairwise identity by state. In total, 241 samples (70 with first-degree familial relationships and 171 with second-degree familial relationships) were removed. The rest samples were tested for population stratification with the principal components stratification method, resulting in the exclusion of 137 population outliers. Finally, a total of 2,743 leprosy patients and 3,573 controls (706 cases and 1,223 controls in GWAS1, 840 cases and 924 controls in GWAS2 and 1,197 cases and 1,426 controls in GWAS3) passed the sample QC filters and were used in subsequent analyses.

Phasing and imputation. The SHAPEIT version 2 (ref. 31) was used to conduct phasing analysis on the basis of the common SNPs, and separately for each ancestry groups. The SNP imputation was carried out with IMPUTE (ref. 32) version 2.2.2 software and 1,000 Genomes Project Phase I reference panel (March 2012 release) in NCBI Build 37 (hg19) coordinates. We subsequently analysed only those SNPs that could be imputed with high confidence (info score $\left.r^{2}>0.8\right)$, had a MAF more than $1 \%$ in all samples and without significant deviation from HWE in the controls $\left(P<1 \times 10^{-5}\right)$. In total, 5,287,069 imputed SNPs and 258,961 genotyped SNPs passed QC and were tested in the association analysis.

Statistical analysis. We first performed the association analysis in three independent GWAS datasets separately. All analyses were carried out using SNPTEST version 2.4.1 software ${ }^{33}$. For each dataset, we included the selected principal components as covariates (1.026 for GWAS1, 1.019 for North Han of GWAS2, 0.98 for South Han of GWAS2, 1.052 for the Southern Minority of GWAS2, 1.038 for North Han of GWAS3 and 1.066 for South Han of GWAS3) in the association model to account for population stratification by EigenCorr ${ }^{34}$.

In the discovery stage, We then performed the meta-analysis of the three independent GWAS datasets using the inverse variance method implemented in META (ref. 35) version 1.3.2. The regional association plots for each locus was generated by the online method LocusZoom ${ }^{36}$ version 1.3.

In the validation stages, log-additive association testing of SNPs was performed using PLINK v1.07. The meta-analysis of the combined discovery and validation samples of 8,156 cases and 15,610 controls was performed using a fixed effects model (inverse variance method). Cochran's Q statistics were performed to evaluate the significance of heterogeneity among individual studies and Bonferronicorrected heterogeneity $P$ values of $<0.05$ were considered significant.

To check whether additional independent association existed within the identified loci, conditional logistic repression analyses were performed by either SNPTEST (Stage 1) or PLINK (Stage 2 and 3).

Genotyping and quality control of the selected SNPs in the validation stage. To validate novel signals identified by the discovery analysis, we applied two strategies to select the potential independent associations. The top SNPs from independent novel loci that showed suggestive association at $P<5 \times 10^{-4}$ in the newly added discovery sample (GWAS 3 ), or in the meta-analysis results of the combined discovery datasets (GWAS $1+2+3$ ) were selected.

Genotyping of the two independent validation stages was conducted using the Sequenom MassARRAY system (Agena Bioscience, Shanghai, China) and TaqMan custom genotyping assays on a 7900 HT Fast Real-Time PCR System (Applied Biosystems, Foster City, CA, USA) according to the manufacturers' instructions. In total, 167 SNPs were selected for stage 2, and 21 were selected for stage 3 using these methods. Of the 21 SNPs in Stage 3, four SNPs failed in genotyping analysis, as they were rejected in the design process (three SNPs) or had a bad genotyping cluster (one SNP). Therefore, they were re-genotyped by using TaqMan custom genotyping assays. In validation analysis, we removed SNPs with a call rate $<90 \%$ or undetermined clusters, and samples with call rate $<95 \%$. 
HLA imputation. We imputed dense SNPs, as well as classical HLA alleles (HLA-A, HLA-B, HLA-C, HLA-DQA1, HLA-DQB1, HLA-DRB1, HLA-DPA1 and HLA-DPB1) and coding variants across the HLA region (chr6: 29.0-33.0 Mb, hg19) in the discovery stage studies using SNP2HLA (ref. 37). Imputation was based on a reference panel from the Pan-Asian ${ }^{38}$ array and consisted of genotypes from individuals of Asian descent who were typed for classical HLA 4-digit alleles. The log-additive regression model was used to perform association analysis for all the variants. We used the principal components identified in each discovery data set to correct for population stratification. The final results were generated by fixed effects model meta-analysis.

Gene annotation and prioritization. The gene prioritization strategies were based on two methods: (1) differential gene expression analysis, which estimated the relative gene expression difference between leprosy biopsy and healthy control skin through an unpublished RNA-sequence dataset (27 leprosy biopsies versus 18 healthy controls). The genes in closest physical proximity to the lead variant were taken into consideration as potentially causal. (2) eQTL analyses were based on the annotation tool HaploReg v4.1, which accounts for the effect of SNPs on expression from multiple eQTL studies. SNPs with an LD $r^{2}>0.9$ and $D^{\prime}>0.9$ were also considered.

Heritability and enrichment analyses. For the heritability analysis, we used the method that was implemented in the GCTA software ${ }^{39}$ to estimate the contribution of common SNPs. The genetic similarity matrix was estimated using all genotyped autosomal SNPs with a MAF of $>0.05$ in all the discovery datasets. We used the default option (restricted maximum likelihood, REML) to fit the appropriate variance components model. We assumed that the leprosy prevalence as 0.0001 to estimate the heritability on the liability scale. We also conducted the heritability partitioning of leprosy GWAS by tissue and functional category using the LD score regression.

Data availability. The genome-wide association SNP results are available upon request by contacting F.Z at zhangfuren@hotmail.com. Any additional data (beyond those included in the main text and Supplementary Information) that support the findings of this study are also available from the corresponding author upon request.

\section{References}

1. Britton, W. J. \& Lockwood, D. N. J. Leprosy. Lancet 363, 1209-1219 (2004).

2. World Health Organization. WHO Expert Committee on Leprosy. World Health Organization Technical Report Series 1-61, 1-61 (2012)

3. Mira, M. T. et al. Susceptibility to leprosy is associated with PARK2 and PACRG. Nature 427, 636-640 (2004).

4. Moraes, M. O. et al. Interleukin-10 promoter single-nucleotide polymorphisms as markers for disease susceptibility and disease severity in leprosy. Genes Immun. 5, 592-595 (2004).

5. Roy, S. et al. Association of vitamin D receptor genotype with leprosy type. J. Infect Dis. 179, 187-191 (1999)

6. Hagge, D. A. et al. Lymphotoxin-alpha and TNF have essential but independent roles in the evolution of the granulomatous response in experimental leprosy. Am. J. Pathol. 174, 1379-1389 (2009).

7. da Silva, S. A. et al. HLA-DR and HLA-DQ alleles in patients from the south of Brazil: markers for leprosy susceptibility and resistance. BMC Infect. Dis. 9, 134 (2009)

8. Zhang, F.-R. et al. Genomewide association study of leprosy. N. Engl. J. Med. 361, 2609-2618 (2009).

9. Zhang, F. et al. Identification of two new loci at IL23R and RAB32 that influence susceptibility to leprosy. Nat. Genet. 43, 1247-1251 (2011).

10. Liu, H. et al. Identification of IL18RAP/IL18R1 and IL12B as leprosy risk genes demonstrates shared pathogenesis between inflammation and infectious diseases. Am. J. Hum. Genet. 91, 1-7 (2012).

11. Liu, H. et al. An association study of TOLL and CARD with leprosy susceptibility in Chinese population. Hum. Mol. Genet. 22, 4430-4437 (2013).

12. Liu, H. et al. Discovery of six new susceptibility loci and analysis of pleiotropic effects in leprosy. Nat. Genet. 47, 267-271 (2015).

13. Zeggini, E. et al. Meta-analysis of genome-wide association data and large-scale replication identifies additional susceptibility loci for type 2 diabetes. Nat. Genet. 40, 638-645 (2008).

14. Voight, B. F. et al. Twelve type 2 diabetes susceptibility loci identified through large-scale association analysis. Nat. Genet. 42, 579-589 (2010).

15. Manning, A. K. et al. A genome-wide approach accounting for body mass index identifies genetic variants influencing fasting glycemic traits and insulin resistance. Nat. Genet. 44, 659-669 (2012).

16. Lee, H. J. et al. Association study of polymorphisms in synaptic vesicleassociated genes, SYN2 and CPLX2, with schizophrenia. Behav. Brain Funct. 1, 15 (2005).
17. Mirnics, K., Middleton, F. A., Marquez, A., Lewis, D. A. \& Levitt, P. Molecular characterization of schizophrenia viewed by microarray analysis of gene expression in prefrontal cortex. Neuron 28, 53-67 (2000).

18. Adams, A. E., Rosenblatt, M. \& Suva, L. J. Identification of a novel parathyroid hormone-responsive gene in human osteoblastic cells. Bone 24, 305-313 (1999).

19. Vernon, E. G. et al. The parathyroid hormone-responsive B1 gene is interrupted by a $\mathrm{t}(1 ; 7)(\mathrm{q} 42 ; \mathrm{p} 15)$ breakpoint associated with Wilms' tumour. Oncogene 22, 1371-1380 (2003).

20. Nouh, M. A. et al. Cathepsin B: a potential prognostic marker for inflammatory breast cancer. J. Transl. Med. 9, 1 (2011).

21. Niedergethmann, M. et al. Angiogenesis and cathepsin expression are prognostic factors in pancreatic adenocarcinoma after curative resection. Int. J Pancreatol. 28, 31-39 (2000).

22. Büth, H. HaCaT keratinocytes secrete lysosomal cysteine proteinases during migration. Eur. J. Cell. Biol. 83, 781-795 (2004).

23. Lee, Y.-C. et al. Two new susceptibility loci for Kawasaki disease identified through genome-wide association analysis. Nat. Genet. 44, 522-525 (2012).

24. Saito, T., Kitayama, D., Sakamoto, A., Tsuruoka, N. \& Arima, M. Effective collaboration between IL-4 and IL-21 on B cell activation. Immunobiology 213, 545-555 (2008).

25. Rani, R., Fernandez-Vina, M. A., Zaheer, S. A., Beena, K. R. \& Stastny, P. Study of HLA class II alleles by PCR oligotyping in leprosy patients from north India Tissue Antigens 42, 133-137 (1993).

26. Singh, M., Balamurugan, A., Katoch, K., Sharma, S. K. \& Mehra, N. K. Immunogenetics of mycobacterial infections in the North Indian population. Tissue Antigens 69, 228-230 (2007)

27. Schauf, V. et al. Leprosy associated with HLA-DR2 and DQwl in the population of northern Thailand. Tissue Antigens 26, 243-247 (1985).

28. Vanderborght, P. R. et al. HLA-DRB1 ${ }^{\star} 04$ and $\mathrm{DRB} 1^{\star} 10$ are associated with resistance and susceptibility, respectively, in Brazilian and Vietnamese leprosy patients. Genes Immun. 8, 320-324 (2007).

29. Alcina, A. et al. Multiple sclerosis risk variant HLA-DRB1 ${ }^{\star} 1501$ associates with high expression of DRB1 gene in different human populations. PLOS ONE 7, e29819 (2012).

30. Tsuchiya, N., Kawasaki, A., Tsao, B. P. \& Komata, T. Analysis of the association of HLA-DRB1, TNFa promoter and TNFR2 (TNFRSF1B) polymorphisms with SLE using transmission disequilibrium test. Genes Immunol. 2, 317-322 (2001)

31. Delaneau, O., Zagury, J.-F. \& Marchini, J. Improved whole-chromosome phasing for disease and population genetic studies. Nat. Methods 10, 5-6 (2013).

32. Howie, B. N., Donnelly, P. \& Marchini, J. A flexible and accurate genotype imputation method for the next generation of genome-wide association studies. PLoS Genet. 5, e1000529 (2009).

33. Marchini, J., Howie, B., Myers, S., McVean, G. \& Donnelly, P. A new multipoint method for genome-wide association studies by imputation of genotypes. Nat. Genet. 39, 906-913 (2007).

34. Lee, S., Wright, F. A. \& Zou, F. Control of population stratification by correlation-selected principal components. Biometrics 67, 967-974 (2011).

35. Liu, J. Z. et al. Meta-analysis and imputation refines the association of $15 \mathrm{q} 25$ with smoking quantity. Nat. Genet. 42, 436-440 (2010).

36. Pruim, R. J. et al. LocusZoom: regional visualization of genome-wide association scan results. Bioinformatics 26, 2336-2337 (2010).

37. Jia, X. et al. Imputing amino acid polymorphisms in human leukocyte antigens. PLoS ONE 8, e64683 (2013).

38. Pillai, N. E. et al. Predicting HLA alleles from high-resolution SNP data in three Southeast Asian populations. Hum. Mol. Genet. 23, 4443-4451 (2014).

39. Yang, J., Lee, S. H., Goddard, M. E. \& Visscher, P. M. GCTA: a tool for genomewide complex trait analysis. Am. J. Hum. Genet. 88, 76-82 (2011).

\section{Acknowledgements}

We thank the individuals who participated in this project and Shandong Computer Science Center (National Supercomputer Center in Jinan), which provided us with the platform for statistical analysis. This work was funded by grants from the National Natural Science Foundation of China $(81472869,81402593,81573036,81502736$, 81620108025), the National Clinical Key Project of Dermatology and Venereology, the Shandong Provincial Independent Innovation Project (ZR2015HZ001), Shandong Province independent innovation and achievement transformation project (2014CGZH1307), the Shandong Provincial Advanced Taishan Scholar Construction Project, the Innovation Project of Shandong Academy of Medical Science, the Natural Science Foundation of Shandong Province (ZR2013HQ041, ZR2014YL044, BS2015YY042, 2014ZRC03145, ZR2015PH027, ZR2015YL035, ZR2015PH040), the Shandong Provincial Medical and Health Development Project (2014GSF118001, 2014WS0064)

\section{Author contributions}

F.Z. conceived of this study and obtained the financial support. F.Z. and H.L. designed the study. H.L., Q.Y., X.F., F.B. undertook recruitment and collected phenotype data 
H.L., Z.W., G.Y., X.F., C.W., Y.X., F.B., Z.Y., J.L., L.S., Y.Y., M.C., Z.M., H.W., P.H., Q.Y., N.W., J.C. conducted sample selection and performed the genotyping of all samples. T.D., W.Y., Y.X., H.X., J.Y., J.L., P.S., G.N., X.C., X.W., X.C. contributed to DNA extraction and clinical data collection. Z.W., Y.S, Y.L., A.I., J.L. undertook data checking, statistical analysis and bioinformatics analyses. H.L. was responsible for sample selection, genotyping and project management. Y.S. and Z.W. wrote the first draft. All authors contributed to the final manuscript, with F.Z., L.H., Z.W., Y.S., X.F. and G.Y. playing the key roles.

\section{Additional information}

Supplementary Information accompanies this paper at http://www.nature.com/ naturecommunications

Competing financial interests: The authors declare no competing financial interests.

Reprints and permission information is available online at http://npg.nature.com/ reprintsandpermissions/
How to cite this article: Wang, Z. et al. A large-scale genome-wide association and metaanalysis identified four novel susceptibility loci for leprosy. Nat. Commun. 7, 13760 doi: 10.1038/ncomms13760 (2016).

Publisher's note: Springer Nature remains neutral with regard to jurisdictional claims in published maps and institutional affiliations.

(c) (i) This work is licensed under a Creative Commons Attribution 4.0 International License. The images or other third party material in this article are included in the article's Creative Commons license, unless indicated otherwise in the credit line; if the material is not included under the Creative Commons license, users will need to obtain permission from the license holder to reproduce the material. To view a copy of this license, visit http://creativecommons.org/licenses/by/4.0/

(C) The Author(s) 2016 